\title{
Electronic Pulses from Pulsed Field Emission of CNT Cathodes
}

\author{
Xianqi Wei $\left(\mathbb{D},{ }^{1,2,3}\right.$ Xiaoli Wang, ${ }^{1}$ Xin Li $\left(\mathbb{D},{ }^{1,4}\right.$ and Weihua Liu ${ }^{1}$ \\ ${ }^{1}$ Department of Microelectronics, School of Electronics and Information Engineering, Xian Jiaotong University, \\ Xi'an, Shaanxi 710049, China \\ ${ }^{2}$ Science School, Huaihai Institute of Technology, Lianyungang, Jiangsu 222005, China \\ ${ }^{3}$ Research Institute of Xian Jiaotong University, No. 328 Wenming Road, Xiaoshan District, Hangzhou City, \\ Zhejiang Province 311215, China \\ ${ }^{4}$ Guangdong Shunde Xian Jiaotong University Academy, No. 3 Deshengdong Road, Daliang, Shunde District, \\ Foshan City, Guangdong Province 528300, China
}

Correspondence should be addressed to Xianqi Wei; wei.wxq@163.com

Received 14 November 2017; Revised 17 January 2018; Accepted 26 February 2018; Published 16 April 2018

Academic Editor: Haihui Ruan

Copyright (C) 2018 Xianqi Wei et al. This is an open access article distributed under the Creative Commons Attribution License, which permits unrestricted use, distribution, and reproduction in any medium, provided the original work is properly cited.

\begin{abstract}
We presented a demonstration of infrared laser irradiated field emission electronic pulse based on carbon nanotube (CNT) cathodes. Electronic pulses greatly depended on pulsed infrared laser and were almost synchronous with laser pulses. We have designed a pulsed field emission cathode based on CNTs and investigated correlation between electronic pulse and laser pulse, acquiring the shortest width of electronic pulses about $50 \mathrm{~ms}$ and turn-on field less than $0.14 \mathrm{~V} / \mu \mathrm{m}$. Besides, we have studied the thermal effect on the pulsed field emission of CNT cathodes caused by laser heating. Interestingly, the thermal effect has caused an enhancement of emission current but resulted in a waveform distortion on short electronic pulses. The application of laser pulses on CNT cathodes would extend conventional electron sources to a pulsed electron source and offered a possibility of pulsed field emission. These results were encouraging us to prepare further studies of ultrafast electronic pulses for high-frequency electron sources.
\end{abstract}

\section{Introduction}

In the applications of ultrafast electron microscopy, free electron laser, satellite communication, picosecond cathodoluminescence, particle accelerators and $\mathrm{THz}$ vacuum microelectronics, and electron sources are limited to ultrafast electron pulses with the stringent requirements of high-power and high-frequency [1-7]. However, with the advent of electron beam from Nanometer-scale metallic tip of cathode emitters induced by femtosecond laser, conventional continuous electron sources are gradually replaced by ultrafast pulsed electron sources [8-13]. Sharp metallic tip of cathode emitters irradiated by laser pulses has represented a feasibility and implementation of the method for realizing ultrafast pulsed electron sources in recent years [14]. With the generation of pulsed electron source, much attention has been paid to explore cathode emitters of pulsed field emission in the related subjects, such as electron rescattering at sharp metallic tips, emission spectrum of laser-matter interaction, coherent field emission images, laser acceleration of relativistic electrons, and emission mechanism [15-19]. Although conventional metallic nanotip of cathode emitters takes advantage of an electric field enhancement both in the static and optical electric field, it is certainly worth considering that the complicated process of manufacture and thermal ablation of metallic nanotips served as cathode emitters of pulsed field emission. To extend the studies on conventional metallic nanotip of cathode emitters, we studied the pulsed electron cathode emitters in a popular material of carbon nanotubes (CNTs).

The sharp apex of individual CNTs is an intrinsic property as a nanomaterial, while conventional metallic tips, for example, tungsten, gold, molybdenum, or copper, were produced by electrochemical etching [16-19]. Contrasting to the tip apex of individual CNTs, the diameter of metallic tips is much larger and has to employ a complicated manufacturing process. The nanometer-sized tip apex of CNTs and their high aspect ratio result in a strong field enhancement at the sharp tip of cathode emitter. Besides, another key advantage 


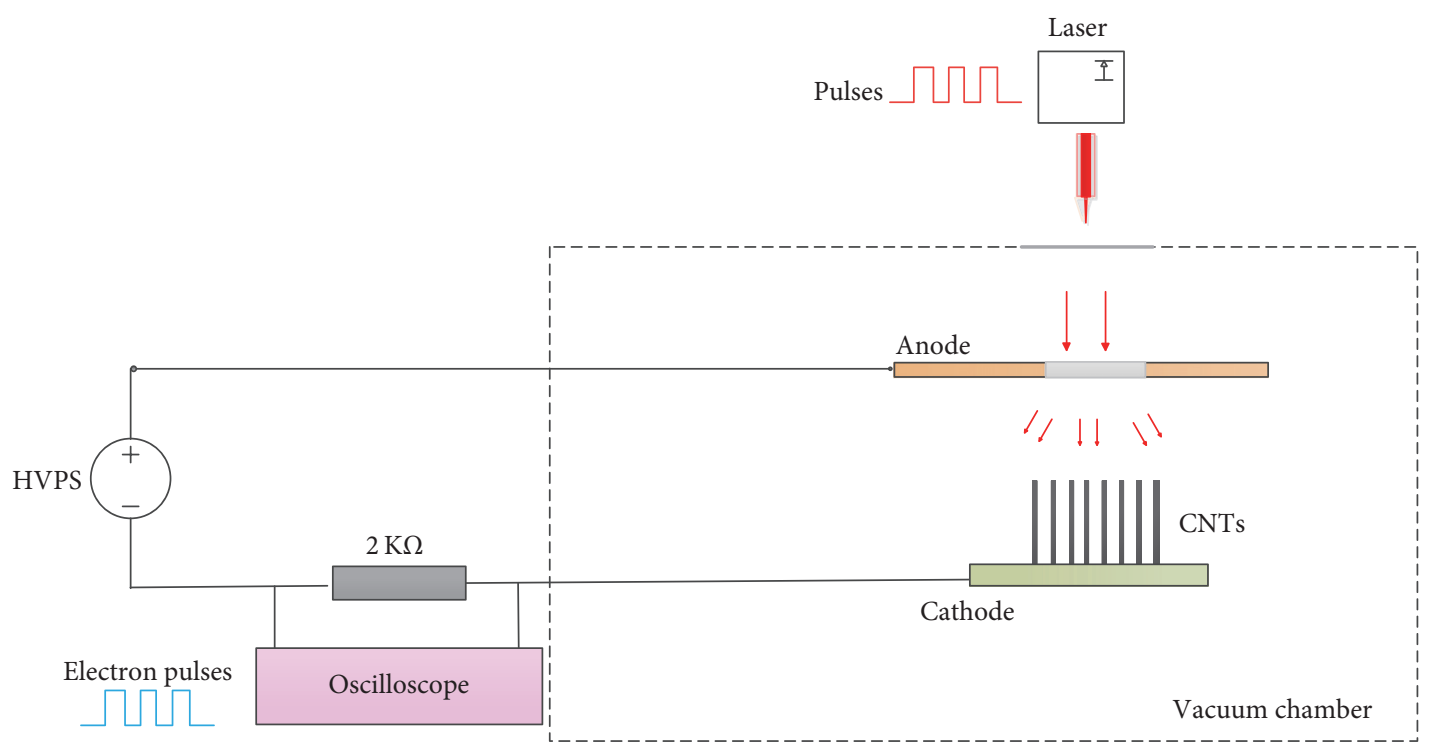

Figure 1: Schematic diagram of pulsed field emission system experimental set-up based on carbon nanotube (CNT) cathode emitters.

of CNTs is that of providing a new method to control surface morphology of the cathode emitters by doping with other elements on the growth process [20]. And CNTs are a desired flexibility material with the convenient and simply preparation process, which has started to receive significant interest in application of flexible display, flexible sensor, radio frequency identification, bullet-proof vest, spacesuit, wearable devices, and other smart electronic devices in comparison with metallic material [21-24]. Capitalizing on the atomic/macroscopic duality of structure of the nanotip of cathode emitters, CNTs can be modeled from an atomic point of view, unlike conventional metallic tip of cathode emitters which can only be modeled in bulk [19], giving great help to theoretical research. In this paper, we have designed a pulsed field emission system in which CNT cathodes are irradiated by pulsed laser and investigated the property of emission electronic pulses so as to gain insight into the pulsed field emission mechanism for preparing ultrafast pulsed electron source.

\section{Experimental}

CNTs were synthesized on $\mathrm{SiO}_{2}$ substrate by chemical vapor deposition (CVD) using iron phthalocyanine. The detailed information about the growth of CNTs was reported in our previous research [25]. Field emission tests were performed in a vacuum chamber with pressure about $5 \times 10^{-5} \mathrm{~Pa}$. A DC bias voltage was applied between the anode and cathode to establish a static electric field by Stanford Research Systems MODEL PS325. The emission current was monitored by a Keithley 2000 multimeter or an Oscilloscope TDS 3014B. The cathodes of CNT arrays were irradiated by Ytterbium Fiber Laser to realize the pulsed field emission when the bias voltage was applied. In the experiment, we presented the pulsed field emission system based on photofield emission mechanism. Irradiating CNT cathodes with infrared laser pulses led to pulsed field emission $[10,18,26]$. Figure 1 shows the experimental set-up of pulsed field emission system based on CNT cathodes. The output from an Ytterbium Fiber Laser was focused on the anode plate with $2 \mathrm{~mm}$ spot size and faced the cathode plate in an ultrahigh vacuum chamber of $5 \times 10^{-5} \mathrm{~Pa}$. The laser operated at 1 watt with a center wavelength of $1064 \mathrm{~nm}$ and produced a train of pulse from $50 \mathrm{~ms}$ to $10000 \mathrm{~ms}$ which could be tuned by a control panel. The CNT cathodes were mounted on the cathode plate in the ultrahigh vacuum chamber and located $350 \mu \mathrm{m}$ away from the anode plate. The intensity and width of laser pulse which propagated through anode plate were carefully controlled by the collimation system and control panel. And then the collimated laser was used to irradiate CNT cathodes of area $2 \mathrm{~mm} \times$ $2 \mathrm{~mm}$ to excite electrons by absorbing photons for emitting electronic pulses, when the applied voltage was on.

\section{Results and Discussions}

In field emission, electron emission happens when the tip bias voltage of cathode emitters is sufficiently large for electrons in the vicinity of Femi-level to tunnel through the barrier. By applying a bias voltage to the tip of cathode emitters, a tunneling barrier is formed and tunneling electron emission occurs. In the photofield emission, electrons are excited to an intermediate state by one or several photons absorption and subsequent tunneling through the surface barrier into vacuum by field emission, when a laser irradiates the cathodes $[14,19,26,27]$. Figure 2 shows electronic pulses curve from the pulsed field emission of CNT cathodes, when the width of laser pulses (defined as the pulse-on T1) and off-time interval (defined as the pulse-off T2) are both 10000 ms. Interestingly, the width of electronic pulses is also $10000 \mathrm{~ms}$ or so, almost synchronous with laser pulses. The average amplitude of electronic pulses is $0.027 \mathrm{~mA} / \mathrm{cm}^{2}$ and the fluctuation is less than $20 \%$ on applied voltage of $50 \mathrm{~V}$. More important, the 
TABLE 1: Amplitude $\left(I_{\text {on }}-I_{\text {off }}\right)$ of electronic pulses on applied voltage of $50 \mathrm{~V}, 100 \mathrm{~V}, 400 \mathrm{~V}, 700 \mathrm{~V}$, and $1000 \mathrm{~V}$, respectively. $T 1=T 2=10000 \mathrm{~ms}$.

\begin{tabular}{lccccc}
\hline Voltage $(\mathrm{V})$ & 50 & 100 & 400 & 700 & \\
\hline Amplitude $\left(\mathrm{mA} / \mathrm{cm}^{2}\right) I_{\text {on }}-I_{\text {off }}$ & 0.23 & 0.53 & 1.05 & 0.45 & \\
\hline
\end{tabular}

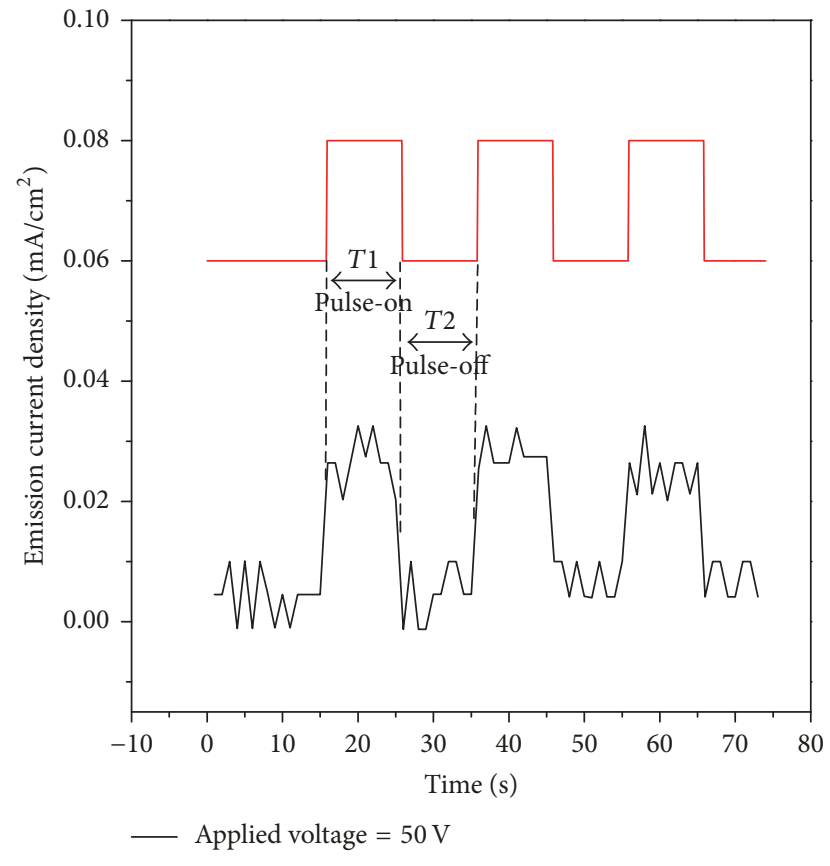

FIGURE 2: Plot of field emission electronic pulses on CNT cathodes irradiated by pulsed laser, on applied voltage of $50 \mathrm{~V}$. Black line represents the emission electronic pulse profile with width of $10000 \mathrm{~ms}(T 1=T 2)$. Red line represents a train of laser pulses with the width of $10000 \mathrm{~ms}$.

emission current density is nearly zero on laser pulse-off with applied voltage of $50 \mathrm{~V}$, proving that electron emissions are excited only on the condition of laser pulse-on $(T 1)$. The preliminary results show the realization of electron emission pulses from CNT cathodes and turn-on electric field less than $0.14 \mathrm{~V} / \mu \mathrm{m}$ (turn-on electric field is defined as the electric field required to produce a current density of $0.01 \mathrm{~mA} / \mathrm{cm}^{2}$ ), which is incomparable with reference to previous studies, such as CNTs on nickel foam $(0.53 \mathrm{~V} / \mu \mathrm{m})$ [28], graphene-CNT $(0.73 \mathrm{~V} / \mu \mathrm{m})$ [29], CNTs on silicon pillars $(2.16 \mathrm{~V} / \mu \mathrm{m})$ [30], and multilayer graphene-CNTs $(0.93 \mathrm{~V} / \mu \mathrm{m})$ [31]. Actually, the turn-on voltage of the CNT cathodes was about $80 \mathrm{~V}$ $(0.23 \mathrm{~V} / \mu \mathrm{m})$ by field emission. On applied voltage of $50 \mathrm{~V}$ $(0.14 \mathrm{~V} / \mu \mathrm{m})$, electron emission would not happen if without laser. This result definitely proved the photofield emission mechanism of which electrons were excited to an intermediate state by laser and subsequently tunneled through barrier into vacuum by field emission.

Figure 3 shows electronic pulses curve from pulsed field emission of CNT cathodes irradiated by laser pulses, on the same working condition of laser pulses except different applied voltage of $100 \mathrm{~V}, 400 \mathrm{~V}, 700 \mathrm{~V}$, and $1000 \mathrm{~V}$, respectively, contrasting to the insert of emission current density of cathodes without laser illumination by field emission without laser illumination. With applied voltage increase, the amplitude $\left(I_{\text {on }}-I_{\text {off }}\right)$ of electronic pulses increases on lower applied voltage of $100 \mathrm{~V}$ and $400 \mathrm{~V}$, as shown in Figures $3(\mathrm{a})$ and 3(b). However, the amplitude decreases with the applied voltage continuously increasing to $700 \mathrm{~V}$ and $1000 \mathrm{~V}$, as shown in Figures 3(c) and 3(d). The corresponding values of amplitude are listed in Table $1 . I_{\text {on }}$ is the average amplitude of emission electronic pulses on electron pulse-on, and $I_{\text {off }}$ is the average valley value on electron pulse-off. The electronic pulses signals appear obviously on low applied voltages, while the pulses cannot be distinguished on the high applied voltage of $1000 \mathrm{~V}\left(I_{\text {on }}-I_{\text {off }} \approx 0\right)$. Interestingly, the amplitude of $I_{\text {on }}-$ $I_{\text {off }}$ increases firstly and then decreases until electronic pulses signals are gradually buried in the DC signal of emission current with applied voltage continuous increase, as showed in Table 1. Practically, the emission current of fabricated CNT cathodes was the result of the combined effect of infrared laser and applied voltage but not the simple sum of emission current caused by photofield emission and field emission. On low applied voltage, photofield emission was dominated in emission process, and applied voltage just narrowed the potential barrier in the vicinity of threshold. On high voltages, emission mechanism was dominated by conventional field emission rather than pulsed field emission. Nevertheless, the laser field instantaneously wiggled the barrier. Emission electrons caused by laser pulses just led to a fluctuation for emission current of the field emission. With the applied voltage continuing to increase, emission electrons caused by laser pulses could be ignored due to being too weak comparing with emission current caused by the field emission. The obtained results helped to understand and realize the emission mechanism of pulsed field emission from CNT cathode.

To increase the frequency of electronic pulses, shortening the period of electronic pulses is one of popular methods for pulsed field emission as an ultrafast pulsed electron source, especially in decreasing width of electronic pulses. Figure 4 shows electronic pulses curve from CNT cathodes irradiated by the laser pulse of $T 1=50 \mathrm{~ms}$, while $T 2$ are $1000 \mathrm{~ms}$ and $100 \mathrm{~ms}$, respectively. The results showed the width of electronic pulses was about $50 \mathrm{~ms}$, and electronic pulse-off was $1000 \mathrm{~ms}$ as shown in Figure 4(a) and $100 \mathrm{~ms}$ in Figure 4(b), respectively. However, pulse broadening was obviously observed as shown in Figure 4(b). For the further study, the response time was investigated, and the results showed the pulse broadening arose from the thermal effect. Thermal effect which came from laser heating up tips of CNT cathodes existed in the pulsed field emission and could enhance the emission current. By virtue of the very fast sampling rate of $2.5 \mathrm{GS} / \mathrm{s}$, oscilloscope was used to detect response time of electronic pulses. Figure 5 shows a single electronic pulse with the laser pulse-on of $T 1=5000 \mathrm{~ms}$ and applied voltage of $400 \mathrm{~V}$. It is found that emission current increases sharply when the laser pulse of $T 1=5000 \mathrm{~ms}$ is on and remarkably drops down when the laser pulse is off. Moreover, the evolution process of emission current is not instantaneous on 


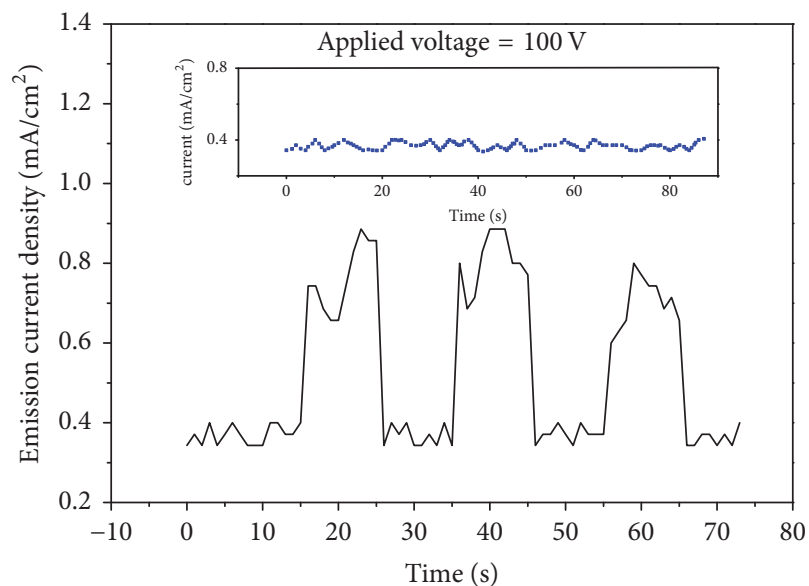

(a)

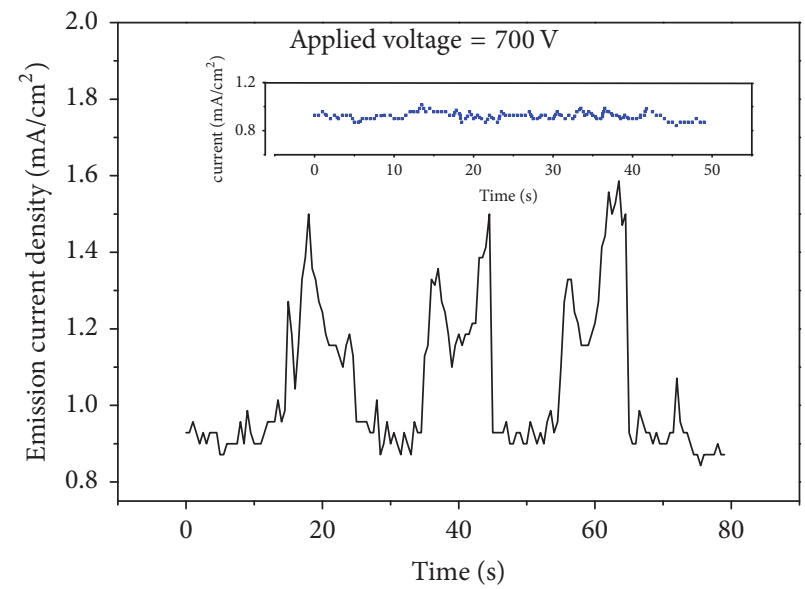

(c)

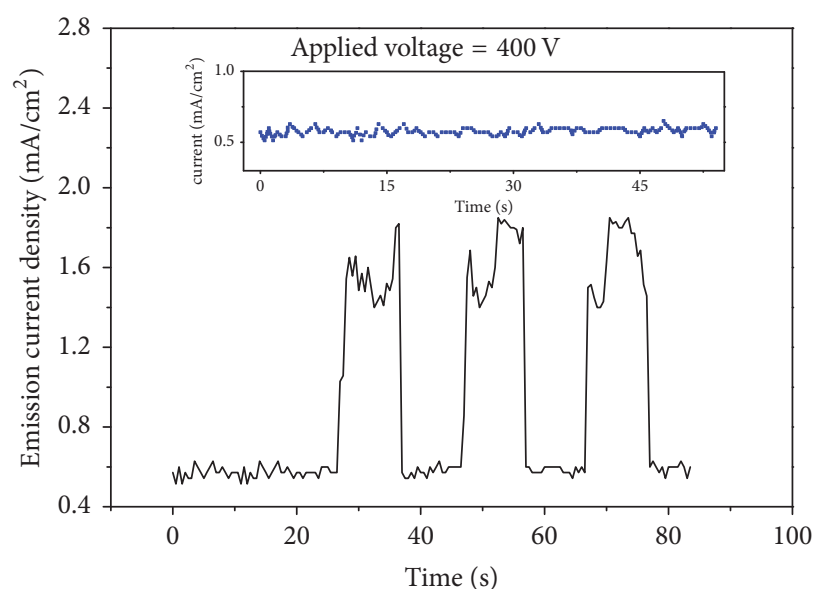

(b)

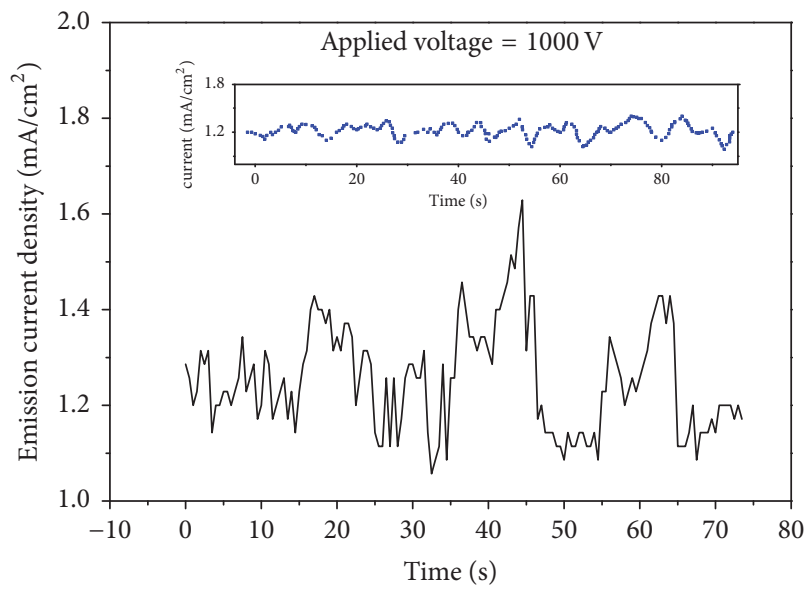

(d)

FIGURE 3: (Black line) Plots of field emission electronic pulses profile on CNT cathodes irradiated by pulsed laser on different applied voltage of $100 \mathrm{~V}, 400 \mathrm{~V}, 700 \mathrm{~V}$, and $1000 \mathrm{~V}$, respectively. The width of emission electronic pulse is $10000 \mathrm{~ms}$. The laser working condition is that the pulse width of $T 1$ and laser pulse-off of $T 2$ are both $10000 \mathrm{~ms}$. The insert (blue squares) is corresponding to the emission current density without laser illumination by field emission.

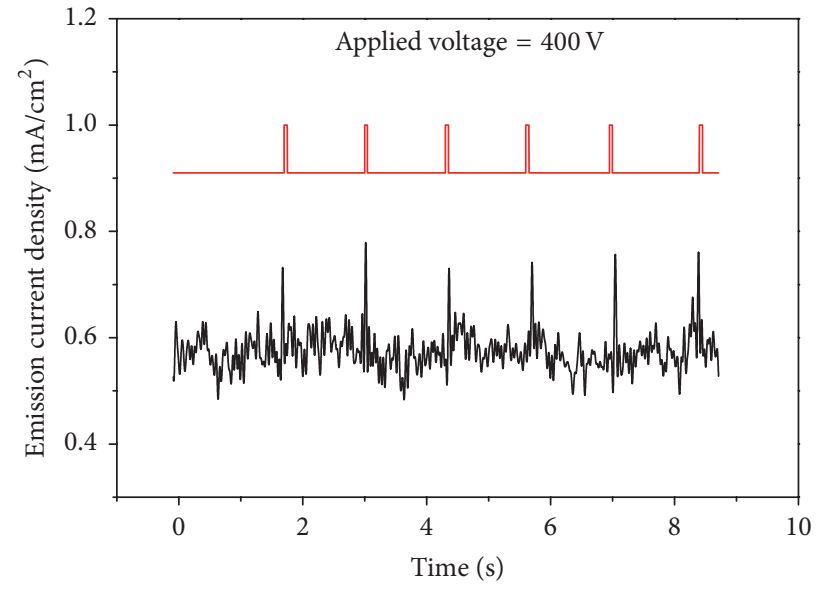

(a)

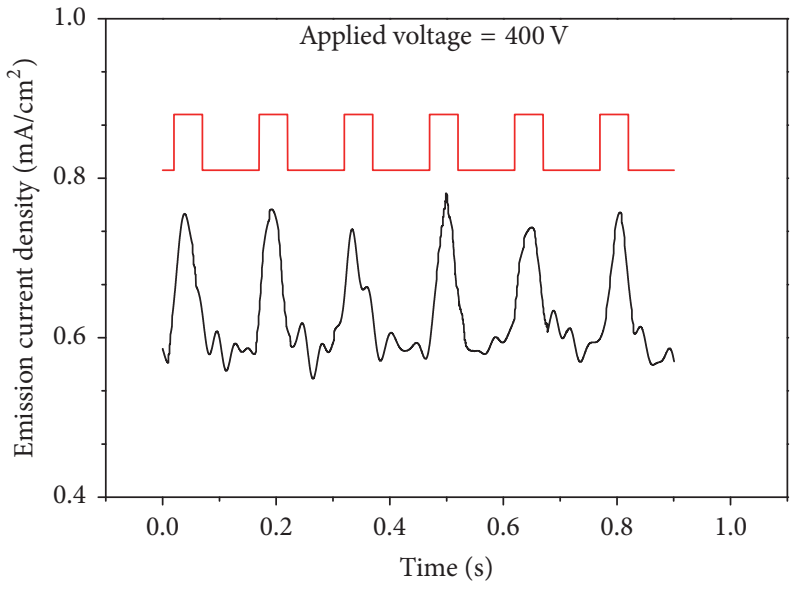

(b)

FIGURE 4: (Black line) Plots of field emission electronic pulses profile with the width of $50 \mathrm{~ms}$ on CNT cathodes irradiated by pulsed laser on $400 \mathrm{~V}(T 1 \neq T 2$ ). (a) $T 1$ width of laser pulses (red line) is $50 \mathrm{~ms}$ and $T 2$ is $1000 \mathrm{~ms}$. (b) $T 1$ width is $50 \mathrm{~ms}$ and $T 2$ is $100 \mathrm{~ms}$. 


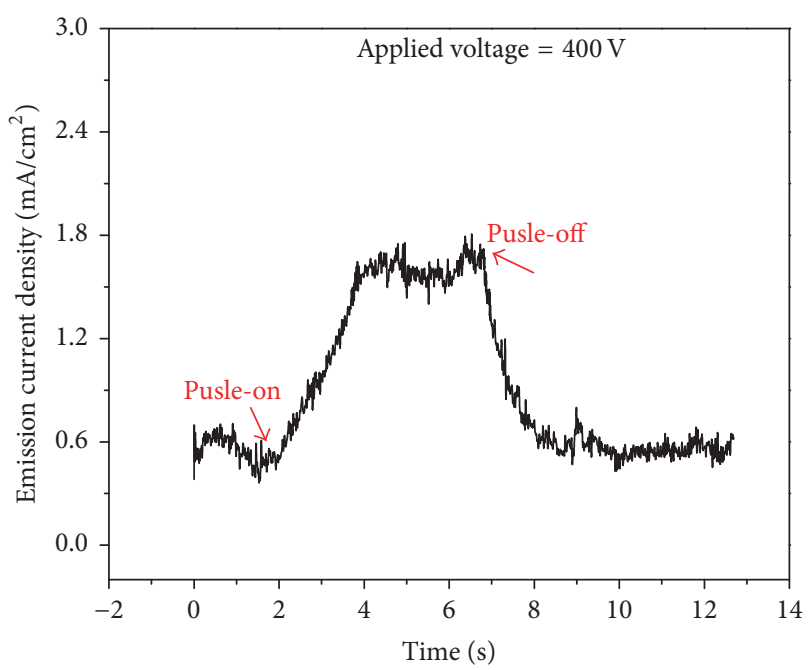

FIGURE 5: Plot of one period of electronic pulse from pulsed field emission of CNT cathodes irradiated by pulsed laser of $400 \mathrm{~V}(T 1=$ $5000 \mathrm{~ms}, \mathrm{~T} 2=10000 \mathrm{~ms}$ ).

laser pulse-on and pulse-off. The rise time of emission current jumping to plateau takes $2 \mathrm{~s}$ or so when laser pulse is on, and the fall time spends more than $2 \mathrm{~s}$ when laser pulse is off. Besides, the amplitude is much higher than that of value in Figure 4 on the same applied voltage $400 \mathrm{~V}$. Working conditions of the electronic pulse in Figure $4(T 1=50 \mathrm{~ms} ; T 2=$ $1000 \mathrm{~ms}, 100 \mathrm{~ms})$ and Figure $5(T 1=5000 \mathrm{~ms} ; T 2=10000 \mathrm{~ms})$ are mainly associated with $T 1$ and $T 2$ of laser pulses on the same applied voltage, but the amplitudes are quite different. Irradiating of the CNT cathodes by laser pulses heated the tips and caused the apex electron temperature to evolve periodically in time [9], so thermally enhanced field emission increased drastically obeying laser excitation and caused the pulse broadening.

A major reason for pulse broadening was thermal effect which played an important role in the field emission mechanism, especially in short electronic pulses occurring. In Figure 5, the electronic pulse had enough time to reach to the saturation value on laser pulse-on of $T 1=5000 \mathrm{~ms}$. However, electronic pulses were compelled to drop down before reaching to the saturation value on the short laser pulse-on of $T 1=50 \mathrm{~ms}$ in Figure 4(b) and compelled to rise up before dropping to the valley value on condition of $T 2=100 \mathrm{~ms}$. After repeatedly verifying, the optimum working condition of laser pulse irradiated CNT cathodes was that the laser pulse-off $T 2$ was greater than or equal to 2 times the laser pulse-on $T 1$ for realizing ultrafast electronic pulses. These results showed that thermal effect enhanced the pulsed field emission of CNT cathodes irradiating by pulsed infrared laser and resulted in a pulse broadening especially on short electronic pulses.

\section{Conclusion}

Irradiating CNT cathodes with laser pulses led to pulsed field emission. We investigated the emission electronic pulses from pulsed field emission of carbon nanotube (CNT) cathodes by tuning the pulsed laser field and attained the short electronic pulses of $50 \mathrm{~ms}$ and turn-on field below $0.14 \mathrm{~V} / \mu \mathrm{m}$. With the applied voltage increase, electronic pulses amplitude increased firstly and then decreased till it was gradually buried in the emission current. With the width of electronic pulses decrease, pulse broadening existed in the pulsed field emission caused by thermal effect which played an important role in the field emission mechanism, especially in short electronic pulses occurring. To reduce the electronic pulse distortion, the optimum working condition of laser pulse irradiated CNT cathodes was $T 2 \geq T 1$ for realizing short electronic pulses. These results extended conventional electron sources and provided a possibility of realizing ultrashort electronic pulses as a high-frequency electron source.

\section{Conflicts of Interest}

The authors declare that they have no conflicts of interest.

\section{Acknowledgments}

This work was financially supported by grants from the National Natural Science Foundation of China (nos. 91123018, 51625504, and 61671368), Shaanxi Natural Science Foundation (2014JM7277), Science and Technology Planning Project of Zhejiang Province, China (2017C31087), and Science and Technology Planning Project of Guangdong Province, China (2017A010103004).

\section{References}

[1] V. Ortalan and A. H. Zewail, "4D scanning transmission ultrafast electron microscopy: single-particle imaging and spectroscopy," Journal of the American Chemical Society, vol. 133, no. 28, pp. 10732-10735, 2011.

[2] A. Adhikari, J. K. Eliason, J. Sun, R. Bose, D. J. Flannigan, and O. F. Mohammed, "Four-dimensional ultrafast electron microscopy: insights into an emerging technique," ACS Applied Materials \& Interfaces, vol. 9, no. 1, pp. 3-16, 2017.

[3] R. Bormann, S. Strauch, S. Schäfer, and C. Ropers, "An ultrafast electron microscope gun driven by two-photon photoemission from a nanotip cathode," Journal of Applied Physics, vol. 118, no. 17, Article ID 173105, pp. 1108-1116, 2015.

[4] J. P. Hall, F. E. Poynton, P. M. Keane et al., "Monitoring one-electron photo-oxidation of guanine in DNA crystals using ultrafast infrared spectroscopy," Nature Chemistry, vol. 7, no. 12, pp. 961967, 2015.

[5] H. Yoneda, Y. Inubushi, K. Nagamine et al., "Atomic inner-shell laser at 1.5-ångström wavelength pumped by an X-ray free-electron laser," Nature, vol. 524, no. 7566, pp. 446-449, 2015.

[6] C. Behrens, F.-J. Decker, Y. Ding et al., "Few-femtosecond timeresolved measurements of X-ray free-electron lasers," Nature Communications, vol. 5, pp. 3762-3769, 2014.

[7] J. Duris, P. Musumeci, M. Babzien et al., "High-quality electron beams from a helical inverse free-electron laser accelerator," Nature Communications, vol. 5, pp. 4928-4935, 2014.

[8] B. D. Patterson, R. Abela, H.-H. Braun et al., "Coherent science at the SwissFEL x-ray laser," New Journal of Physics, vol. 12, pp. 035012-035029, 2010. 
[9] C. Kealhofer, S. M. Foreman, S. Gerlich, and M. A. Kasevich, "Ultrafast laser-triggered emission from hafnium carbide tips," Physical Review B: Condensed Matter and Materials Physics, vol. 86, no. 3, Article ID 035405, pp. 165-173, 2012.

[10] C. Ropers, D. R. Solli, C. P. Schulz, C. Lienau, and T. Elsaesser, "Localized multiphoton emission of femtosecond electron pulses from metal nanotips," Physical Review Letters, vol. 98, no. 4, pp. 043907-043912, 2007.

[11] J. Hoffrogge, J. Paul Stein, M. Krüger et al., "Tip-based source of femtosecond electron pulses at $30 \mathrm{keV}$," Journal of Applied Physics, vol. 115, no. 9, pp. 094506-094515, 2014.

[12] W. Luo, T. P. Yu, M. Chen et al., "Generation of bright attosecond $\mathrm{X}$-ray pulse trains via Thomson scattering from laser-plasma accelerators," Optics Express, vol. 22, pp. 32098-32106, 2014.

[13] Y. Jiang, L. C. Liu, H. M. Müller-Werkmeister et al., "Femtosecond electron diffraction study of the spin crossover dynamics of single crystal [Fe(PM-AzA)2](NCS)2," Ultrafast Phenomena XIX, vol. 162, pp. 283-286, 2015.

[14] M. Krüger, M. Schenk, M. Förster, and P. Hommelhoff, "Attosecond physics in photoemission from a metal nanotip," Journal of Physics B: Atomic, Molecular and Optical Physics, vol. 45, no. 7, pp. 074006-074012, 2012.

[15] T. Qi, L. Dong, Y. Qiao et al., "Enhanced electron field emission of $\mathrm{Cu}$ implanted microcrystalline diamond films after annealing," Vacuum, vol. 134, pp. 141-149, 2016.

[16] X. Q. Yang, Y. Hu, J. L. Zhang, Y. Q. Wang, C. M. Pei, and F. Liu, "Preparation of boron nanowires using AuPd nanoparticles as catalyst and their field emission behavios," Acta Physica Sinica, vol. 63, pp. 048102-048191, 2014.

[17] S. Tsujino, F. Le Pimpec, J. Raabe et al., "Static and optical field enhancement in metallic nanotips studied by two-photon photoemission microscopy and spectroscopy excited by picosecond laser pulses," Applied Physics Letters, vol. 94, no. 9, pp. 093508093512, 2009.

[18] B. Piglosiewicz, S. Schmidt, D. J. Park et al., "Carrier-envelope phase effects on the strong-field photoemission of electrons from metallic nanostructures," Nature Photonics, vol. 8, no. 1, pp. 37-42, 2014.

[19] M. R. Bionta, B. Chalopin, A. Masseboeuf, and B. Chatel, "First results on laser-induced field emission from a CNT-based nanotip," Ultramicroscopy, vol. 159, pp. 152-155, 2015.

[20] B. A. Kakade, V. K. Pillai, D. J. Late et al., "High current density, low threshold field emission from functionalized carbon nanotube bucky paper," Applied Physics Letters, vol. 97, no. 7, pp. 073102-073105, 2010.

[21] P. Ilanchezhiyan, A. S. Zakirov, G. M. Kumar et al., "Highly efficient CNT functionalized cotton fabrics for flexible/wearable heating applications," RSC Advances, vol. 5, no. 14, pp. 1069710702, 2015.

[22] S. Huang, C. Zhao, W. Pan, Y. Cui, and H. Wu, "Direct writing of half-meter long CNT based fiber for flexible electronics," Nano Letters, vol. 15, no. 3, pp. 1609-1614, 2015.

[23] Z. Ahmad, K. S. Karimov, and F. Touati, "Flexible impedance and capacitive tensile load Sensor based on CNT composite," Chinese Physics B, vol. 25, no. 2, pp. 028801-028806, 2016.

[24] R. Kumar, R. K. Singh, D. P. Singh, A. R. Vaz, R. R. Yadav, and C. S. Rout, "Synthesis of self-assembled and hierarchical palladium-CNTs-reduced graphene oxide composites for enhanced field emission properties," Mater \& Design, vol. 122, pp. 110-117, 2017.
[25] X. Wei, Y. Zhu, X. Xia, X. Wang, W. Liu, and X. Li, "Carbon nanotube cathodes covered on the cylindrical surface of a fiber," RSC Advances, vol. 5, no. 22, pp. 17049-17053, 2015.

[26] P. G. Chavan, S. S. Badadhe, I. S. Mulla, M. A. More, and D. S. Joag, "Synthesis of single crystalline CdS nanocombs and their application in photo-sensitive field emission switches," Nanoscale, vol. 3, no. 3, pp. 1078-1083, 2011.

[27] P. Hommelhoff, Y. Sortais, A. Aghajani-Talesh, and M. A. Kasevich, "Field emission tip as a nanometer source of free electron femtosecond pulses," Physical Review Letters, vol. 96, no. 7, Article ID 077401, pp. 077401-077405, 2006.

[28] M. Song, P. Xu, L. Han et al., "Enhanced field-emission performance from carbon nanotube emitters on nickel foam cathodes," Journal of Electronic Materials, vol. 45, no. 4, pp. 2299-2304, 2016.

[29] J.-H. Deng, G.-A. Cheng, R.-T. Zheng et al., "Catalyst-free, selfassembly, and controllable synthesis of graphene flake-carbon nanotube composites for high-performance field emission," Carbon, vol. 67, pp. 525-533, 2014.

[30] H. S. Uh and S. Park, "Improved field emission properties from carbon nanotubes grown onto micron-sized arrayed silicon pillars with pyramidal bases," Diamond and Related Materials, vol. 54, no. 1, pp. 74-78, 2015.

[31] J.-H. Deng, B. Yu, G.-Z. Li et al., "Self-assembled growth of multi-layer graphene on planar and nano-structured substrates and its field emission properties," Nanoscale, vol. 5, no. 24, pp. 12388-12393, 2013. 


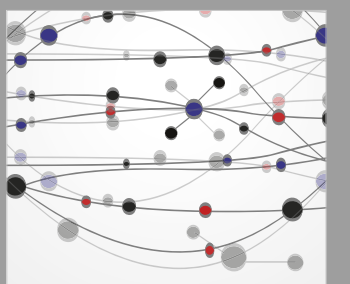

The Scientific World Journal
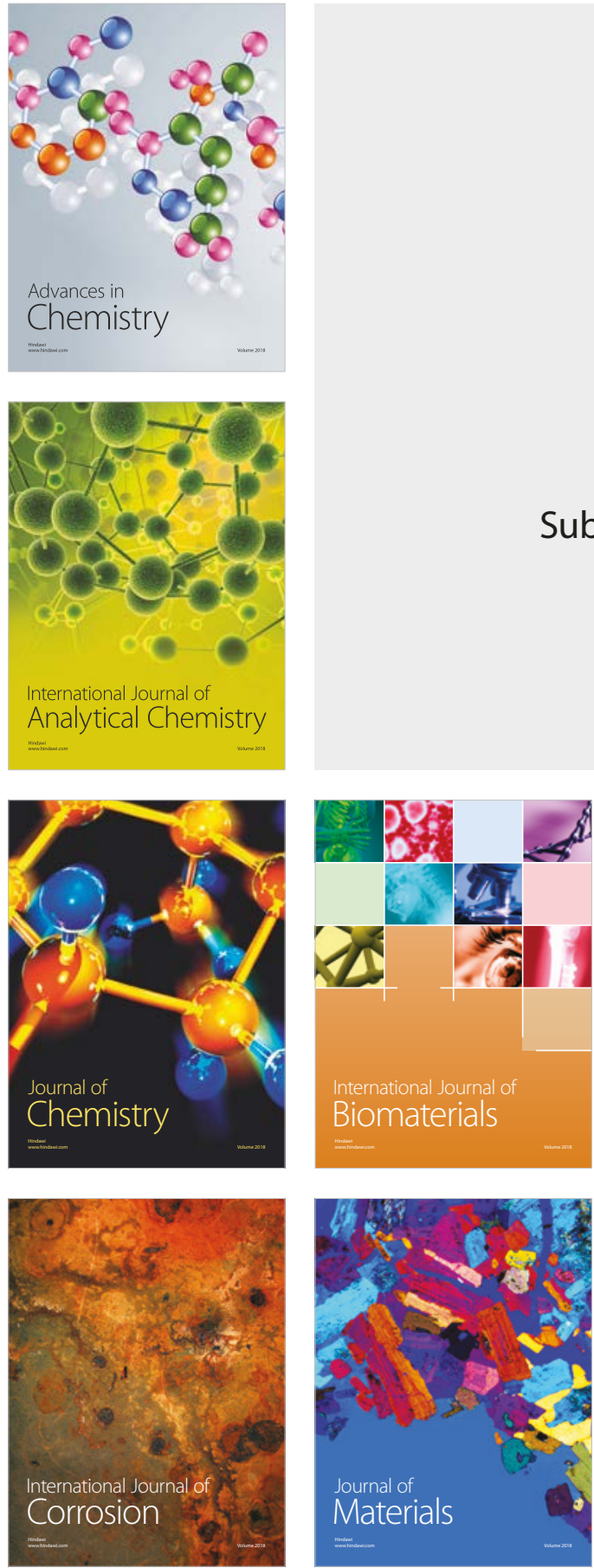

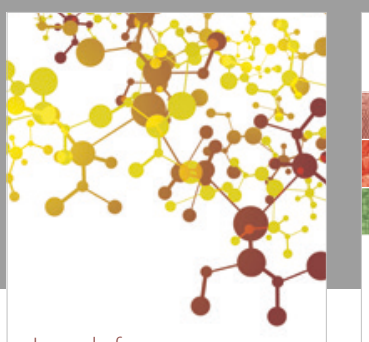

Journal of

Applied Chemistry
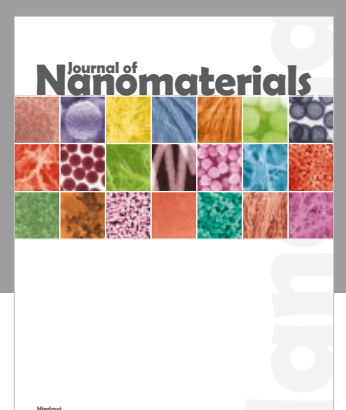

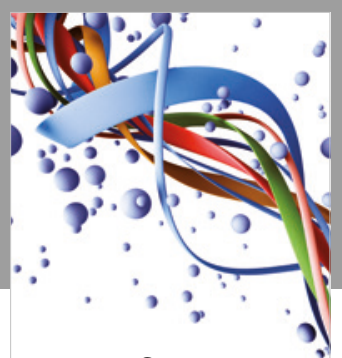

Scientifica

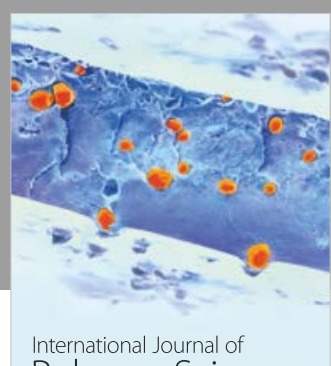

Polymer Science

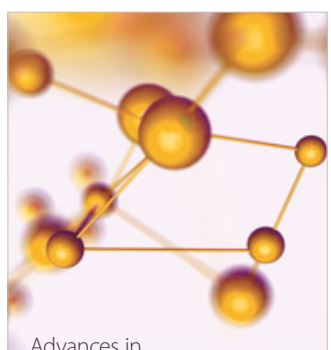

Physical Chemistry
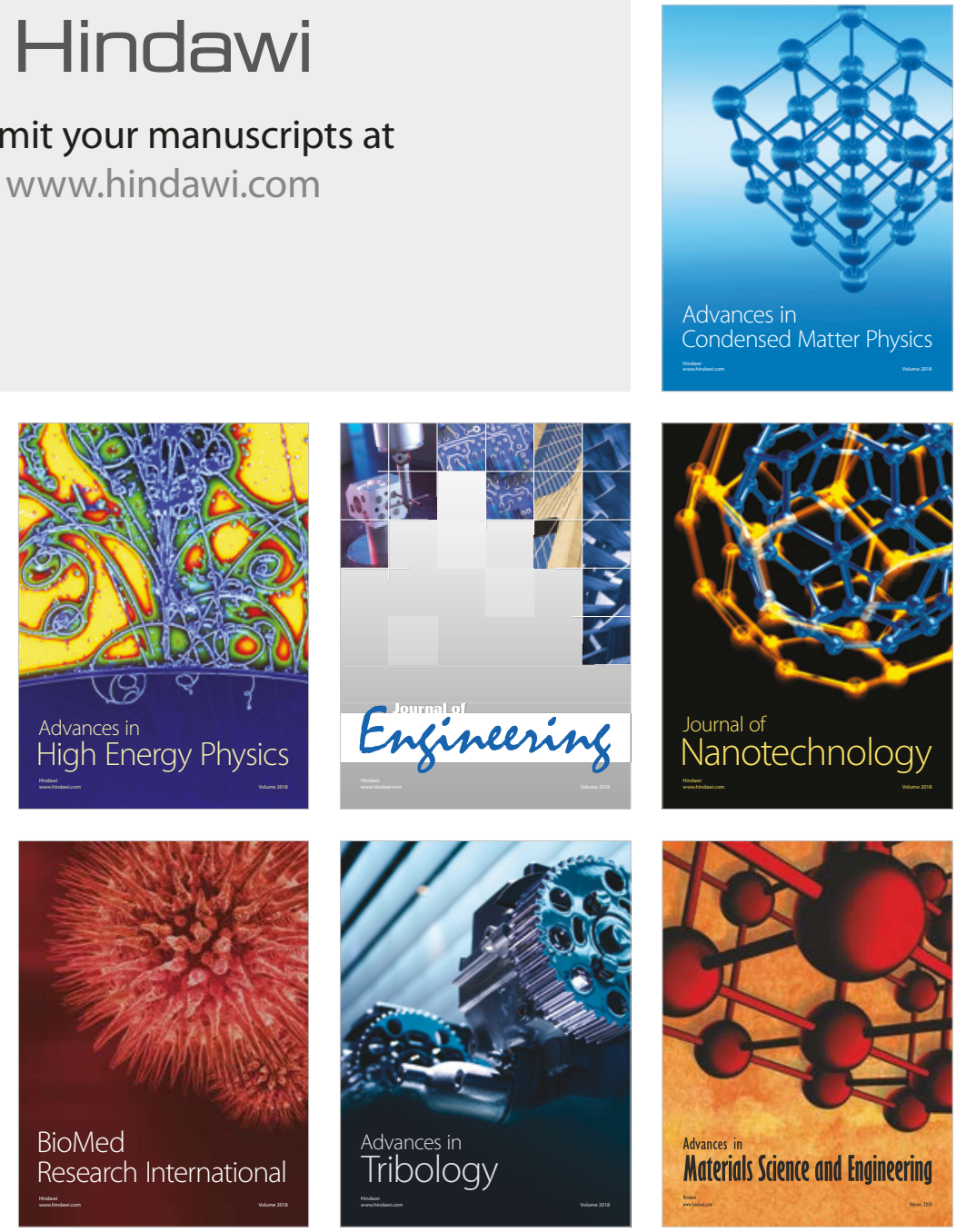